The Astrophysical Journal, 610:1169-1181, 2004 August 1

(C) 2004. The American Astronomical Society. All rights reserved. Printed in U.S.A.

\title{
DO ANOMALOUS COSMIC RAYS MODIFY THE TERMINATION SHOCK?
}

\author{
V. FlorinsKi AND G. P. ZANK \\ Institute of Geophysics and Planetary Physics, University of California, Riverside, CA 92521; vflorins@citrus.ucr.edu, zank@ucrac1.ucr.edu \\ J. R. JOKIPII \\ Lunar and Planetary Laboratory, University of Arizona, Tucson, AZ 85721; jokipii@lpl.arizona.edu \\ AND \\ E. C. Stone and A. C. Cummings \\ Space Radiation Laboratory, California Institute of Technology, Pasadena, CA 91125; ecs@srl.caltech.edu, ace@srl.caltech.edu \\ Received 2004 February 18; accepted 2004 April 14
}

\begin{abstract}
This work extends our previous two-dimensional self-consistent model of the cosmic rays interacting with the solar wind to include anomalous cosmic rays. As before, energetic particles are described kinetically using a Parker equation. The model includes diffusion, convection, and drift effects, as well as shock and compression acceleration and expansion cooling by nonuniform solar wind flow. A new numerical model has been developed featuring an adaptive-mesh refinement algorithm to accommodate small diffusive length scales of low-energy shock-accelerated particles. We show that anomalous cosmic rays have only a minor effect on the termination shock during the time near solar minima. Specifically, cosmic-ray gradients cause the subshock to move away from the Sun by about 1 AU with its compression ratio decreasing by about 5\% compared to the reference case without cosmic-ray effects. We also study the effect of solar wind slowdown by charge exchange downstream of the termination shock, producing compressive flow in this region and resulting in additional acceleration of anomalous cosmic rays in the heliosheath. For the first time, spectra calculated with our self-consistent model show a good agreement with the cosmic-ray data from the two Voyager spacecraft, giving more confidence in the model predictions than the previous parametric studies.
\end{abstract}

Subject headings: acceleration of particles — cosmic rays — interplanetary medium — shock waves — solar wind

\section{INTRODUCTION}

Anomalous hydrogen ions, although essentially absent at the Earth's orbit, have been identified, and their spectra measured, by the two Voyager spacecraft now approaching the termination shock (TS; Christian et al. 1988, 1995; Cummings \& Stone 1998). Their origin is generally thought to be interstellar hydrogen atoms that are ionized by charge exchange with the solar wind protons. Because these pickup ions (PUIs) have significantly higher random velocities than the solar wind protons, they are more easily accelerated to energies at which diffusive shock acceleration by the heliospheric TS becomes effective (Pesses et al. 1981). Anomalous cosmic rays (ACRs) have since been the subject of numerous papers studying their acceleration and propagation in the heliosphere (e.g., Jokipii 1986; Potgieter \& Moraal 1988; Jokipii et al. 1993; Steenberg \& Moraal 1996; Sreenivasan \& Fichtner 2001; see also a very detailed review by Fichtner 2001).

At the same time, the question whether or not ACRs are capable of modifying the TS has not, in our opinion, been answered convincingly. The possible dynamic effects of the diffusive highly energetic plasma component on the solar wind and the TS have been studied extensively in one dimension (Ko \& Webb 1988; Ko et al. 1988; Lee \& Axford 1988; Donohue \& Zank 1993; Chalov \& Fahr 1997; Banaszkiewicz \& Ziemkiewicz 1997). The basic effects are summarized in Lee (1997) and Zank (1999) and consist of a formation of an ACR-mediated shock precursor and an increase in the sub- shock stand-off distance. Multidimensional effects were studied by Fahr et al. (2000), who included ACRs as a separate plasma component produced from the PUI component that, in turn, was produced from charge exchange with interstellar neutrals. Nevertheless, the above studies were largely parametric and the effects predicted ranged from no modification to complete smoothing of the shock structure, depending on ACR intensity (usually related to the injection rate) and the strength of coupling to the thermal plasma, i.e., the diffusion coefficient. A proper treatment of the problem was also hindered by using the hydrodynamic approximation for the energetic particles. As discussed in Florinski et al. (2003), this approach relies on a momentum-averaged diffusion coefficient closure, resulting in the problem being overdetermined. In addition, a fluid approach does not yield particle spectra that can be compared with the available observational data to validate model assumptions.

A one-dimensional kinetic approach to the problem (le Roux \& Fichtner 1997) allowed a direct comparison of model ACRaccelerated spectra and the Voyager data. Their results allowed an estimate of the amount of shock modification attained for fairly realistic energy-dependent diffusion coefficients. This approach was extended to two dimensions by Florinski (2001). The introduction of a second dimensional variable allows the inclusion of drift effects in the model, and these are quite important for perpendicular shocks such as the TS, as demonstrated by Jokipii (1982).

In this paper we study the self-consistent problem of the ACR acceleration at the solar wind TS and its subsequent 
mediation in the framework of a two-dimensional local heliospheric model. In a preceding paper (Florinski \& Jokipii 1999), we discussed the possible effects of galactic cosmic rays (GCRs) in the region downstream of the shock, using a two-dimensional self-consistent model. As before, the present model only includes the region of the heliosphere facing the interstellar flow, since the interstellar flow is not included and an external boundary is placed before the heliopause. Even with this limitation, the model is useful in estimating the extent of shock modification because it allows for a much more detailed diffusion formalism, which includes drift effects.

This paper incorporates several enhancements to our previous model. The cosmic-ray model now includes anomalous particles, and the PUI momentum and energy are now contained in the solar wind equations. Including the ACRs, with their small diffusive length scales, prompted the development of an adaptive-mesh numerical code that increases resolution by an order of magnitude at the shock. In addition, we use a more realistic heliospheric model with latitudinal variations in the wind velocity and number density, and a revised diffusion model (Zank et al. 1996a, 1998; le Roux et al. 1999). We directly compare ACR spectra calculated from the model with in situ measurements made by the two Voyager spacecraft during the previous (positive) solar minimum (Cummings et al. 2002).

\section{SOLAR WIND MODEL}

The new heliospheric model is a descendant of our previous two-dimensional GCR model reported in Florinski \& Jokipii (1999). A number of enhancements have been made to accommodate the ACR injection and acceleration. The model now includes PUIs produced by charge exchange as part of the solar wind plasma. We note that this does not mean that the PUIs become thermalized with the background solar wind (see Isenberg 1986 for a detailed discussion), but only that we do not keep track of the two species individually. Within the confines of the model, we assume that PUIs are isotropized instantaneously and comove with the solar wind. Consequently, multifluid and one-fluid solar wind plasma models are dynamically indistinguishable. We do keep track of the PUI density to model ACR injection; see $\S 3$.

In terms of the hydrodynamic description of the plasma system, cosmic rays, being tenuous, carry no mass or momentum, but only energy. In a spherical $(r-\theta)$ coordinate system with the $z$-axis aligned with the solar rotation axis, the conservation equations for the plasma are

$$
\begin{gathered}
\frac{\partial \rho_{p}}{\partial t}+\frac{1}{r^{2}} \frac{\partial\left(\rho_{p} u_{p} r^{2}\right)}{\partial r}+\frac{1}{r \sin \theta} \frac{\partial\left(\rho_{p} v_{p} \sin \theta\right)}{\partial \theta}=0, \\
\frac{\partial\left(\rho_{p} u_{p}\right)}{\partial t}+\frac{1}{r^{2}} \frac{\partial\left(\rho_{p} u_{p}^{2} r^{2}\right)}{\partial r}+\frac{1}{r \sin \theta} \frac{\partial\left(\rho_{p} u_{p} v_{p} \sin \theta\right)}{\partial \theta}= \\
\frac{\rho_{p} v_{p}^{2}}{r}-\frac{\partial p_{g}}{\partial r}-\frac{\partial p_{c}}{\partial r}+Q_{p-\mathrm{H}}^{u}, \\
\frac{\partial\left(\rho_{p} v_{p}\right)}{\partial t}+\frac{1}{r^{2}} \frac{\partial\left(\rho_{p} u_{p} v_{p} r^{2}\right)}{\partial r}+\frac{1}{r \sin \theta} \frac{\partial\left(\rho_{p} v_{p}^{2} \sin \theta\right)}{\partial \theta}= \\
-\frac{\rho_{p} u_{p} v_{p}}{r}-\frac{1}{r} \frac{\partial p_{g}}{\partial \theta}-\frac{1}{r} \frac{\partial p_{c}}{\partial \theta}+Q_{p-\mathrm{H}}^{v}
\end{gathered}
$$

$$
\begin{gathered}
\frac{\partial}{\partial t}\left[\frac{\rho_{p}\left(u_{p}^{2}+v_{p}^{2}\right)}{2}+\frac{p_{g}}{\gamma-1]}\right. \\
+\frac{1}{r^{2}} \frac{\partial}{\partial r}\left\{u_{p} r^{2}\left[\frac{\rho_{p}\left(u_{p}^{2}+v_{p}^{2}\right)}{2}+\frac{\gamma p_{g}}{\gamma-1}\right]\right\} \\
+\frac{1}{r \sin \theta} \frac{\partial}{\partial \theta}\left\{v_{p} \sin \theta\left[\frac{\rho_{p}\left(u_{p}^{2}+v_{p}^{2}\right)}{2}+\frac{\gamma p_{g}}{\gamma-1}\right]\right\}= \\
-u \frac{\partial p_{c}}{\partial r}-\frac{v}{r} \frac{\partial p_{c}}{\partial \theta}+Q_{p-\mathrm{H}}^{e}-Q_{\mathrm{inj}}+Q_{\mathrm{bnd}}
\end{gathered}
$$

Here $\rho_{p}$ is plasma density, $u_{p}$ and $v_{p}$ are radial and latitudinal plasma velocity components, respectively, $p_{g}$ is the combined thermal pressure of the wind and PUI, and $p_{c}$ is the cosmic-ray pressure. The source terms on the right describe momentum and energy transfer between the cosmic rays and the plasma, as well as charge exchange between the solar wind plasma and the neutral component (various $Q_{p-\mathrm{H}}$ ). The remaining terms in the energy equation describe injection and cooling of the ACRs and are described in $\S 3$.

Our model may be applied only in the upstream direction with respect to the interstellar wind; therefore, we align the $\theta=\pi / 2$ direction of the two-dimensional numerical grid with the heliospheric symmetry axis ("nose" direction). The neutral atoms are thus assumed to have the velocity $\boldsymbol{u}_{\mathrm{H}}$ with components defined by

$$
u_{\mathrm{H}}=-V \sin \theta, v_{\mathrm{H}}=-V \cos \theta,
$$

where we have taken the interstellar wind speed $V=25 \mathrm{~km}$ $\mathrm{s}^{-1}$. For the charge exchange terms we use expressions derived in McNutt et al. (1998). Because it is not possible to include the multidimensional effects of the neutral hydrogen distribution (such as the hydrogen wall) in a local model, we use a fixed distribution of the form (Axford 1972)

$$
n_{\mathrm{H}}=n_{\mathrm{H} \infty} \exp \left[-\frac{r_{0}(\pi-2 \theta)}{2 r \cos \theta}\right]
$$

with $n_{\mathrm{H} \infty}=0.115 \mathrm{~cm}^{-3}$ (Gloeckler et al. 1997) denoting the density of neutral hydrogen inferred for the TS and $r_{0}=$ 4.0 AU. The interstellar neutral $\mathrm{H}$ temperature is taken to be $T=10^{4} \mathrm{~K}$. Despite the simplicity of this approximation, the model is able to correctly capture the deceleration of the solar wind and the reduction of the shock compression ratio $s$.

Our kinematic magnetic field model is the same as in Florinski \& Jokipii (1999) with a modified component $B_{m}$ that Jokipii \& Kota (1989) included in the polar region:

$$
\begin{gathered}
\frac{\partial B_{r}}{\partial t}+\frac{1}{r^{2}} \frac{\partial\left(r^{2} u_{p} B_{r}\right)}{\partial r}+\frac{\partial\left(v_{p} B_{r}\right)}{r \partial \theta}=B_{r}\left(\frac{\partial u_{p}}{\partial r}+\frac{u_{p}}{v_{p}} \frac{\partial u_{p}}{r \partial \theta}\right) \\
\frac{\partial B_{\phi}}{\partial t}+\frac{1}{r} \frac{\partial\left(r u_{p} B_{\phi}\right)}{\partial r}+\frac{\partial\left(v_{p} B_{\phi}\right)}{r \partial \theta}=0 \\
\frac{\partial B_{m}}{\partial t}+\frac{1}{r} \frac{\partial\left(r u_{p} B_{m}\right)}{\partial r}=0
\end{gathered}
$$

The modified magnetic field is assumed to be in the $\theta$ direction (Florinski 2001). The regular $B_{\theta}$ magnetic field may be found 


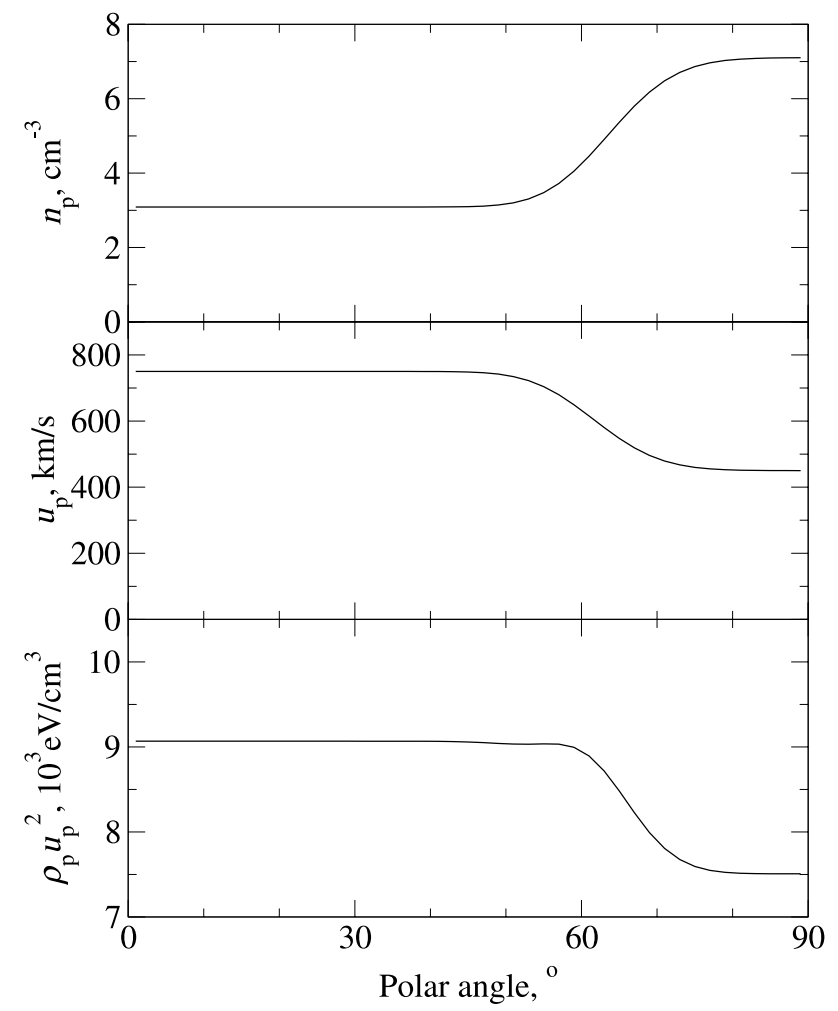

FIG. 1.-Latitudinal variation of the solar wind number density (top), radial velocity (middle), and dynamic pressure (bottom) at 1 AU.

from the steady-state Faraday's law as $B_{\theta}=v_{p} B_{r} / u_{p}$. The average magnitude of the magnetic field is calculated as

$$
B^{2}=B_{r}^{2}+B_{\theta}^{2}+B_{\phi}^{2}+B_{m}^{2}
$$

The supersonic solar wind inflow boundary condition is fixed at $r=r_{0}$, and we specify a fixed confining pressure $p_{g \infty}=1.05 \mathrm{eV} \mathrm{cm}^{-3}$ at the subsonic external boundary. This makes the hydrodynamic problem completely determined. To simulate the different type of wind, we vary the inner boundary conditions with latitude according to Ulysses measurements for the solar minimum (Goldstein et al. 1996); see Figure 1. The fast coronal wind extends between $0^{\circ}$ and $60^{\circ}$ in latitude and has a density of $3.1 \mathrm{~cm}^{-3}$, velocity of $750 \mathrm{~km} \mathrm{~s}^{-1}$, and temperature of $2.6 \times 10^{5} \mathrm{~K}$ at $1 \mathrm{AU}$, while the slow equatorial wind occupies the remaining $30^{\circ}$ and has a density of $7.1 \mathrm{~cm}^{-3}$, velocity of $450 \mathrm{~km} \mathrm{~s}^{-1}$, and temperature of $1.1 \times 10^{5} \mathrm{~K}$ at $1 \mathrm{AU}$. These initial conditions yield a $20 \%$ difference in momentum flux $\rho u^{2}$ between the pole and the equator. We used error functions to create a continuous transition between the polar and the equatorial wind.

Our numerical code (Florinski \& Jokipii 1999) has been modified significantly for this problem. To accommodate the ACRs (see below), a finer resolution was required. To avoid computational overhead, our new model incorporates an adaptive-mesh refinement (AMR) algorithm (Berger \& Colella 1989) to solve the system of equations (1)-(4) and (7)-(9). This method dynamically generates a multigrid hierarchy covering regions with large spatial gradients in plasma density or velocity, such as the TS. The solution on each grid patch is advanced using its own time step that depends on the refinement level $l$ of the patch (i.e., at level 2, grids are advanced

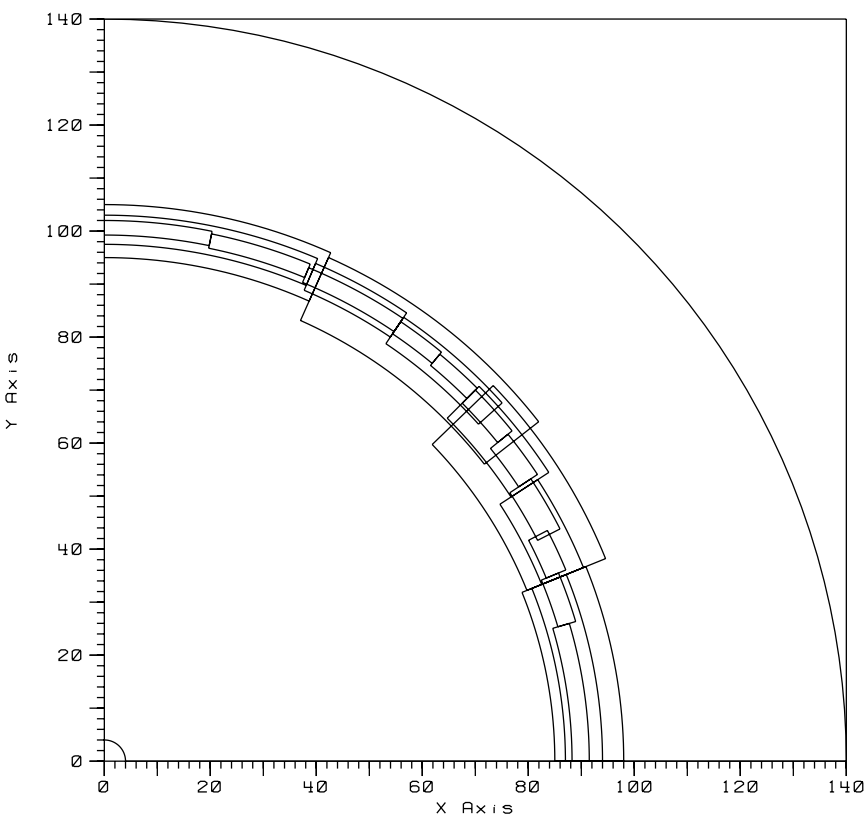

FIG. 2.-Adaptive mesh topology for the simulation described in the text This particular mesh contains one base level, four level 1, six level 2, and 11 level 3 grids.

4 times per single global time step $\Delta t$ ). Communication between grid levels is accomplished through the use of boundary conditions and the update of the coarse grids from overlying finer grids. We use a refinement ratio of two and four grid levels, with the coarsest (level 0) radial grid resolution of $1 \mathrm{AU}$ and the angular resolution of $2^{\circ}$. The number of refinement levels is chosen such that the radial spacing of the finest grid is smaller than the cosmic-ray diffusive length scale of the lowest energy particles, $\Delta r<\kappa_{r r} / u_{p}$, where $\kappa_{r r}$ is the radial diffusion coefficient upstream of the shock (see $\S 3$ ). In this case the finest (level 3) grids have a resolution of $0.125 \mathrm{AU}$ by 0.25 . A typical mesh structure generated by the model is shown in Figure 2. It is worth mentioning that a one-dimensional AMR model including cosmic rays was previously used by Kang et al. (2001) to study cosmic-ray acceleration at a supernova remnant shock.

The advantage of using an AMR technique rather than a uniform refinement is significant. Suppose, for simplicity, that the shock is mostly aligned with $\theta$ grid lines and that the original simulation domain contains $N \times M$ grid cells. Because of the linear nature of the discontinuity, each level of refinement tends to contain approximately the same number of cells $K$ in the radial direction. For a refinement ratio of 2, the total number of cells is estimated to be

$$
N_{\text {cells }}=N M+\sum_{l=1}^{l_{\max }} 2^{l} K M,
$$

where the summation is carried over all refinement levels $l=\left(1 . . l_{\max }\right)$. The total number of time steps needed to complete one global iteration is then

$$
N_{\text {steps }}=1+\frac{K}{N} \sum_{l=1}^{l_{\max }} 2^{2 l} .
$$

For our simulations, typical values are $N=150, M=50$, $K=25$, and $l_{\max }=3$, showing that $N_{\text {cells }}$ is increased by a 
factor of only 3.3 and $N_{\text {steps }}$ by 15 , compared with a simulation on a coarse grid. In contrast, uniform refinement would have resulted in a factor of 64 increase in the number of cells and a factor of 512 increase in computational time. In this case, using AMR is over 30 times more efficient than performing the same computation on a uniform fine mesh.

\section{COSMIC-RAY TRANSPORT}

The cosmic-ray transport equation (Parker 1965; Skilling 1975) can be written in our coordinate system as

$$
\begin{aligned}
& \frac{\partial f}{\partial t}+\left(u_{p}+v_{d, r}\right) \frac{\partial f}{\partial r}+\left(v_{p}+v_{d, \theta}\right) \frac{1}{r} \frac{\partial f}{\partial \theta} \\
& -\frac{1}{r^{2}} \frac{\partial}{\partial r}\left(\kappa_{r r} r^{2} \frac{\partial f}{\partial r}\right)-\frac{1}{r^{2} \sin \theta} \frac{\partial}{\partial \theta}\left(\kappa_{\theta \theta} \sin \theta \frac{\partial f}{\partial \theta}\right) \\
& -\frac{1}{r^{2}} \frac{\partial}{\partial r}\left(\kappa_{\theta r} r \frac{\partial f}{\partial \theta}\right)-\frac{1}{r \sin \theta} \frac{\partial}{\partial \theta}\left(\kappa_{r \theta} \sin \theta \frac{\partial f}{\partial r}\right)= \\
& \frac{1}{3}\left[\frac{1}{r^{2}} \frac{\partial\left(r^{2} u_{p}\right)}{\partial r}+\frac{1}{r \sin \theta} \frac{\partial\left(v_{p} \sin \theta\right)}{\partial \theta}\right] \frac{\partial f}{\partial \ln p}+S_{\text {inj. }}
\end{aligned}
$$

Here $\boldsymbol{v}_{\boldsymbol{d}}$ is the particle drift velocity and $\kappa$ is the (symmetric) diffusion tensor. The last term describes the injection of particles into the acceleration process at the shock. We use an absorbing condition at the upper momentum boundary $p_{\max }$ and at the lower momentum boundary $p_{\min }$ when $\nabla \cdot \boldsymbol{u}<0$. Our choice of a lower boundary condition for $\nabla \cdot \boldsymbol{u}>0$ is discussed later in this section.

Following Florinski (2001) and Florinski et al. (2003) we use the quasi-linear result (Jokipii 1971) for a combined energyinertial power spectrum of slab turbulence, which yields the following expression (Zank et al. 1998) for the diffusion tensor component parallel to the mean field

$$
\kappa_{\|}=\frac{27}{35} \frac{r_{g}^{1 / 3} l_{c}^{2 / 3} w}{A_{\mathrm{sl}}^{2}}\left[\frac{7}{72}\left(\frac{r_{g}}{l_{c}}\right)^{5 / 3}+1\right] .
$$

Here $w$ is the particle velocity, $r_{g}$ is the cyclotron radius, and $l_{c}$ relates to the correlation length of the slab turbulence component that has the amplitude $A_{\mathrm{sl}}^{2}=\left\langle\delta B_{x, \mathrm{sl}}^{2}\right\rangle / B^{2}$, where $\left\langle\delta B_{x, \mathrm{sl}}^{2}\right\rangle$ is the transverse field magnitude of the fluctuations with wavevectors parallel to the mean magnetic field.

Evolution of the incompressible fluctuation spectra (i.e., $A^{2}$ and $l_{c}$ ) and associated diffusion coefficients were studied by Zank et al. (1996a, 1998). Note, however, that their model is not applicable to the hot heliosheath plasma, where compressible fluctuations can become important. Until a more detailed multidimensional turbulence model is developed, we use the same approach as in Florinski \& Jokipii (2003); i.e., we "freeze" the amplitude and the correlation length at the inner boundary and use the same values $A_{\mathrm{sl}}^{2}=0.06$ and $l_{c}=0.03 \mathrm{AU}$ (le Roux et al. 1999) throughout the simulation domain.

Perpendicular diffusion is assumed to be due to magnetic field line wandering, arising mostly from the two-dimensional turbulence component, and is expressible as (Giacalone \& Jokipii 1999; le Roux et al. 1999)

$$
\kappa_{\perp}=a A_{\text {tot }}^{2} \kappa_{\|}
$$

where we use $A_{\mathrm{tot}}^{2}=A_{\mathrm{sl}}^{2}+A_{2 \mathrm{D}}^{2} \simeq 5 A_{\mathrm{sl}}^{2}$ (Bieber et al. 1996), where $A_{2 \mathrm{D}}^{2}$ is the amplitude of two-dimensional fluctuations. Here we use $a=0.1$.

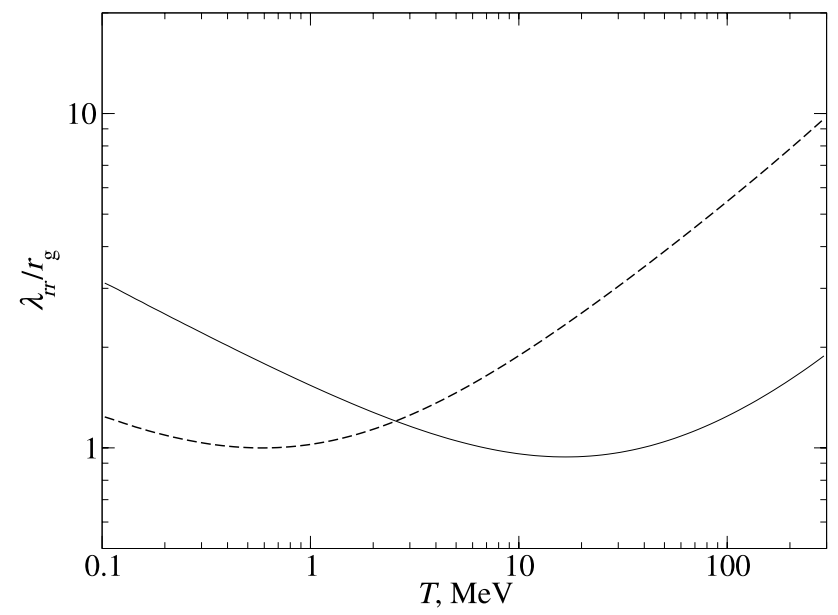

FIG. 3.-Ratio $\lambda_{r r} / r_{g}$ just upstream of the shock at the equator (solid line) and pole (dashed line).

The Parker equation (13) approach to diffusive shock acceleration is valid in the limit of small anisotropy, i.e., applies to high-energy particles. The cosmic-ray streaming flux can be written in component form as

$$
\begin{gathered}
F_{r}=-\frac{4 \pi p^{2}}{w}\left[\kappa_{r r} \frac{\partial f}{\partial r}+\left(\kappa_{r \theta}+\frac{r_{g} w}{3} \frac{B_{\phi}}{B}\right) \frac{1}{r} \frac{\partial f}{\partial \theta}+\frac{u_{p}}{3} \frac{\partial f}{\partial \ln p}\right] \\
F_{\theta}=-\frac{4 \pi p^{2}}{w}\left[\frac{\kappa_{\theta \theta}}{r} \frac{\partial f}{\partial \theta}+\left(\kappa_{r \theta}-\frac{r_{g} w}{3} \frac{B_{\phi}}{B}\right) \frac{\partial f}{\partial r}\right]
\end{gathered}
$$

The total anisotropy is $\xi=3|\boldsymbol{F}| /(4 \pi J)$, where $J=f p^{2}$ is the differential intensity. In the vicinity of the TS the field is almost entirely in the $\phi$ direction, and latitudinal gradients are expected to be small compared with radial gradients in equations (16) and (17). Then, neglecting $\kappa_{r \theta}$ and using a simple approximation for $f(r, p)$ with

$$
\frac{\partial f}{\partial r} \simeq \frac{u f}{\kappa_{r r}}, \frac{\partial f}{\partial \ln p} \simeq-\sigma f,
$$

where $\sigma$ is the power-law index of the particle distribution, we obtain the following expression for the anisotropy:

$$
\xi=\frac{u}{w} \sqrt{(\sigma-3)^{2}+\left(\frac{3 r_{g}}{\lambda_{r r}} \frac{B_{\phi}}{B}\right)^{2}},
$$

where $\lambda_{r r}=3 \kappa_{r r} / w$ is the radial diffusive mean free path. The above expression is equivalent to equation (2.2) of Zank et al. (2001b) with the exception that the latter used a classical scattering limit for the second term under the radical, while we use an unmodified drift velocity expression. At high latitudes the field is dominated by $B_{m}$; hence $B_{\phi} / B \ll 1$, while in the ecliptic region $B_{\phi} / B \sim 1$. The ratio $r_{g} / \lambda_{r r}$ is plotted in Figure 3 . As can be seen from this figure, the radial mean free path exceeds $r_{g}$ at all energies and latitudes. Since for shocks of moderate strength $\sigma \sim 5$, the diffusion approximation is applicable if $w \gg 2 u$ at high latitudes. At low latitudes, $\lambda_{r r} \simeq 3 r_{g}$, giving the condition $w \gg 2.23 u$. These estimates are consistent with Jokipii (1987) and Giacalone et al. (1997). In any case, 
only particles with energies higher than $10 \mathrm{keV}$ can be adequately described by the diffusive model. This does not imply that lower energy particles cannot be accelerated at the shock, only that a model must necessarily take into account the anisotropy of the distribution function to study this process. Monte Carlo simulations (Ellison et al. 1999) show that direct acceleration of PUIs to ACR energies is possible at quasiperpendicular shocks under the assumption of strong isotropic scattering, although this result has not yet been reproduced by models that include self-consistent diffusion.

In our model we set the lower momentum boundary at $100 \mathrm{keV}$ and assume that ions are preaccelerated to the required energy by some other mechanism. Apart from stochastic acceleration (momentum diffusion) by interplanetary turbulence (Chalov et al. 1997), most preacceleration models developed in the literature are variations of the diffusive or drift shock acceleration processes, in which particles gain energy by drifting along the shock front in the $\boldsymbol{u} \times \boldsymbol{B}$ electric field. These include acceleration by traveling interplanetary shocks (Giacalone et al. 1997) and by the TS itself taking into account multiple reflections off the shock front. The latter has been studied by Chalov \& Fahr (1996) and Zank et al. (2001a), using the adiabatic approximation treatment of reflections at an oblique MHD shock and by Zank et al. (1996b) and Lee et al. (1996), who studied ion reflection off the cross-shock electric potential (MRI or "shock surfing"). Note that le Roux et al. (2000) included preacceleration by using a three-stage model in which freshly produced PUIs cooled by the solar wind expansion were first energized by the MRI mechanism and subsequently injected into the diffusive shock acceleration process. At this time we are unable to include preacceleration in the same model because of resolution constraints. Le Roux et al. (2000) found that MRI-accelerated ions produce a shock precursor on the scales of $\ll 0.1 \mathrm{AU}$, which is about an order of magnitude smaller than the finest resolution we have near the shock.

Because PUI distribution at energies appropriate for diffusive shock acceleration is not available in our model, we are required to use an injection term in the cosmic-ray transport equation (13). Here we estimate an injection rate from the PUI number density $n_{i}$ as

$$
S_{\mathrm{inj}}=\frac{\eta}{4 \pi p_{\min }^{2}} n_{i} u_{p} \delta\left(r-r_{s}\right) \delta\left(p-p_{\min }\right)
$$

where $p_{\min }$ is the momentum corresponding to the lower energy limit. The free parameter $\eta$ describes the injection efficiency. It is chosen strictly from the observational considerations; i.e., the computed spectra must be in agreement with the Voyager data. The energy loss term in equation (4), obtained by integrating equation (20), is

$$
Q_{\mathrm{inj}}=\eta T_{\min } m_{p} n_{i} u_{p} \delta\left(r-r_{s}\right),
$$

where $T=p^{2} /\left(2 m_{p}\right)$ is the nonrelativistic particle kinetic energy.

The cosmic-ray pressure that enters into equations (2)-(4) is calculated as

$$
p_{c}=\frac{4 \pi}{3} \int_{p_{\min }}^{\infty} f p^{3} w d p .
$$

We can use infinity as the upper integration limit because the ACR distribution function falls off exponentially at high energies. ACRs, of course, experience cooling in a divergent flow and can, in principle, cool below the minimum injection threshold. These particles are no longer identified as ACRs and instead revert to the solar wind/PUI population. On integrating the cooling term in the transport equation (13) between $p_{\text {min }}$ and $p_{\max }$, where $p_{\max }=p(300 \mathrm{MeV})$ is the upper momentum boundary and $f\left(p_{\max }\right)=0$, one obtains the energy transfer term to the plasma (see eq. [4])

$$
Q_{\mathrm{bnd}}=\frac{4 \pi}{3}(\nabla \cdot u) T_{\min } p_{\min }^{3} f\left(r, p_{\min }\right) .
$$

This approach was used by Zank et al. (1993), but in reverse, i.e., describing injection, rather than cooling. Because cooled particles are lost from the ACR population, no boundary condition of any kind is needed at $p_{\min }$ in divergent flow regions.

We use an absorbing boundary condition at the external radial boundary, located at $150 \mathrm{AU}$. This reflects the fact that diffusion is extremely large in the LISM (Florinski et al. 2003) and that particles can escape freely. The solution at small heliocentric distances is somewhat sensitive to the boundary conditions assumed at the inner radial boundary. Here we use a zero differential streaming flux condition $F_{r}=0$. This assumption is consistent with the $f(r, p)=$ const solution at small $r$ (Fisk et al. 1973). The neutral sheet is treated as narrow, but finite $\left(5^{\circ}\right.$ half-width) by using a multiplier in the drift velocity (Potgieter \& Moraal 1985) throughout the simulation domain.

Finally, while PUI momentum and energy are contained in equations (2)-(4), we calculate their number density separately. This is done strictly for injection purposes using mass conservation

$$
\frac{\partial n_{i}}{\partial t}+\frac{1}{r^{2}} \frac{\partial\left(n_{i} u_{p} r^{2}\right)}{\partial r}+\frac{1}{r \sin \theta} \frac{\partial\left(n_{i} v_{p} \sin \theta\right)}{\partial \theta}=Q_{p-\mathrm{H}}^{d} .
$$

The numerical schemes used to solve equations (1)-(4), (7)(9), (13), and (24) were described in Florinski \& Jokipii (1999) and are carried over to the present model without major changes.

\section{COSMIC-RAY DISTRIBUTION FROM THE MULTIGRID MODEL}

\subsection{Accuracy of the AMR Scheme}

A solution of a hyperbolic system of equations, such as the hydrodynamic system equations (1)-(4), will always approach the accuracy of the fully refined solution because of the interface flux formulation used by finite volume numerical schemes. This cannot, however, be said with the same degree of confidence about the parabolic equation (13). An explicit flux formulation of the cosmic-ray transport equation is very inefficient because of a strict stability constraint on the time step. On the other hand, the implicit nature of an alternated direction implicit (ADI) scheme used here cannot be globally preserved because the solution on each grid patch proceeds independently of the rest of the domain. Nevertheless, as we show below, this scheme still yields a stable solution that converges to the solution obtained on a very fine grid.

For this test we solve the one-dimensional (spherically symmetric) version of equation (13) without drifts. We use the diffusion model described in the previous section with solar wind parameters at $45^{\circ}$ latitude, ignoring wind deceleration by charge exchange. Instead of calculating the solar wind velocity from the model, we specify a uniform $u$ upstream and change to an incompressible flow beyond the shock, located at $80 \mathrm{AU}$. For simplicity, the shock compression ratio is taken to be 4 . 


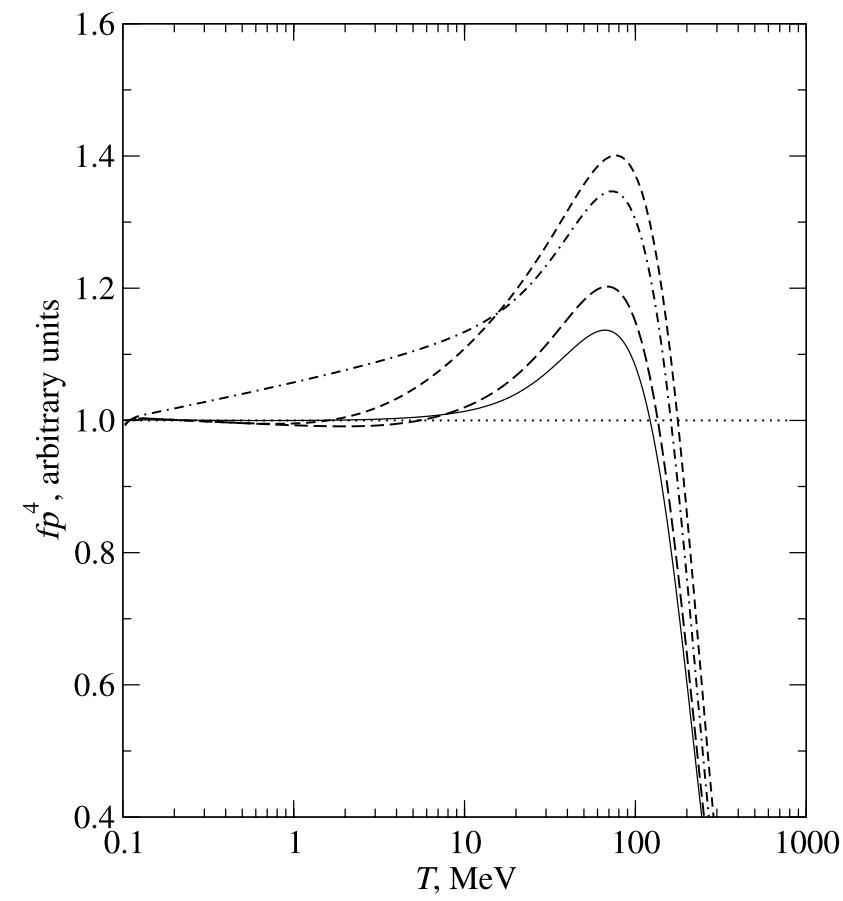

FIG. 4.-ACR spectra at the shock for several different mesh configurations. The solid line shows the solution obtained on a very fine variable grid. The short-dashed line shows the solution obtained on a four-level grid composed of narrow patches with $K=12$, while the long-dashed line shows the result obtained with wide $(K=24)$ patches. The dot-dashed line shows the solution obtained on the coarse grid. The dotted line shows the spectra produced by a strong planar shock.

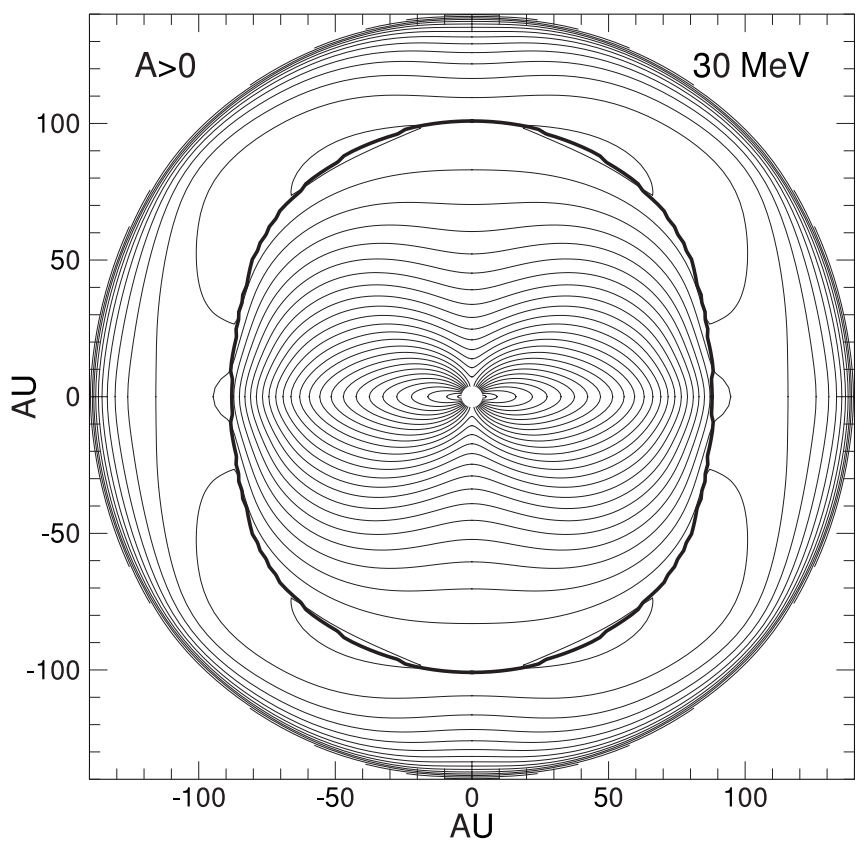

FIG. 5.-The $30 \mathrm{MeV}$ proton intensity for the positive solar minimum. Contours are logarithmically spaced with a $30 \%$ intensity difference between adjacent levels. The TS is shown with a thick solid line. The characteristic maximum at high latitude just downstream of the TS is produced by a combination of drift and shock acceleration effects.
Figure 4 shows the results from the four-level AMR scheme compared with the solution obtained on the coarse grid only and the reference solution (solid line), calculated using a very fine grid. We plot the quantity $f p^{4}$ at $r_{s}$, to emphasize the differences between the models. The general properties of the shock spectra were studied in Florinski \& Jokipii (2003). The spectra consists of three regions: the power law at low energies, cooling-induced cutoff at high energies, and a "bump" in between, owing to a more effective acceleration at these energies.

In this test, the coarse grid resolution was $\Delta r=1 \mathrm{AU}$. Clearly, the coarse solution predicts an incorrect power-law index and should be discarded. The two AMR solutions show the dependence of the accuracy on the patch size. Besides the base grid with $\Delta r=1 \mathrm{AU}$, we used three nested grids, each 12 or 24 cells wide for the two cases, respectively. From Figure 4 one can see that both models produced a correct slope, but the solution on narrow patches significantly overpredicted the height of the bump. The failure of the method for small $K$ stems from the fact that the nested grids do not cover the diffusive length scale of the higher energy cosmic rays, resulting in a loss of accuracy. However, the solution with $K=24$ shows a much better agreement with the reference case. Applying this conclusion to the two-dimensional selfconsistent model, we tuned the grid generation algorithm to produce patches that are relatively wide in the radial direction (see Fig. 2). We judge the accuracy of the AMR method to be acceptable when used in combination with the ADI numerical scheme for the Parker's equation.

\subsection{ACR Intensities and Spectra from the Self-consistent Model}

The spatial distribution of $30 \mathrm{MeV}$ anomalous hydrogen ions is shown in Figures 5 and 6 for the $A>0$ and $A<0$ solar minima conditions, respectively. As expected, the distribution is qualitatively similar to previously published results based on a two-dimensional drift model (Jokipii \& Giacalone 1998). For the positive solar cycle, ACRs drift along the shock toward high latitudes producing the maxima seen in Figure 5.

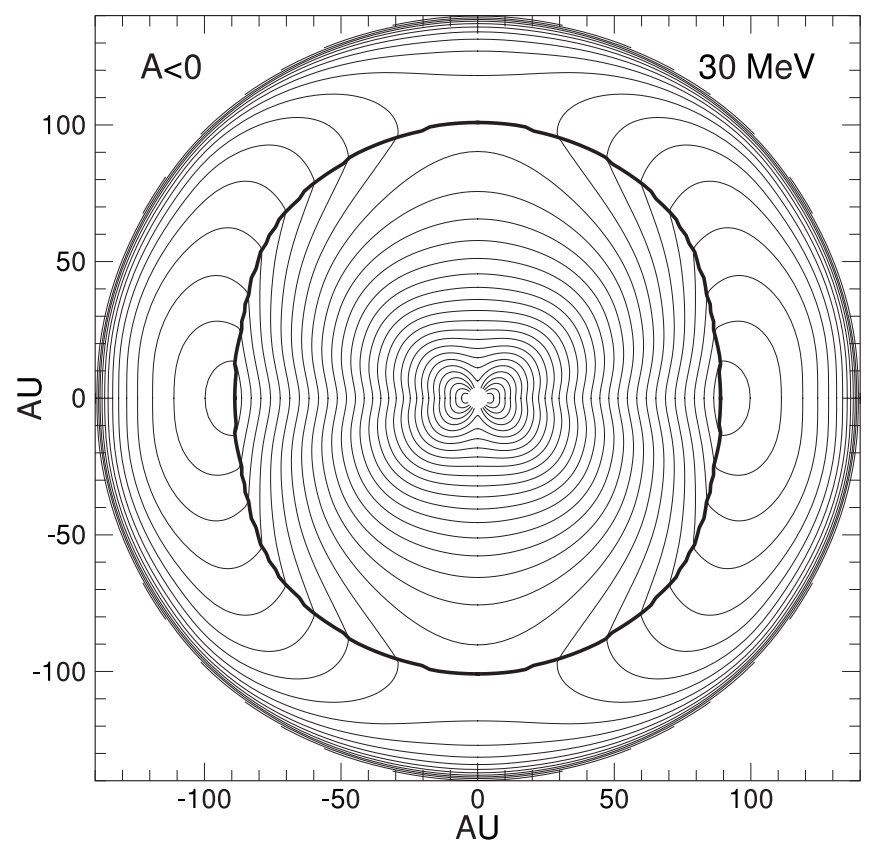

FIG. 6.-Same as Fig. 5, but for the $A<0$ solar minimum. The range of intensity values is the same as in Fig. 5 . 


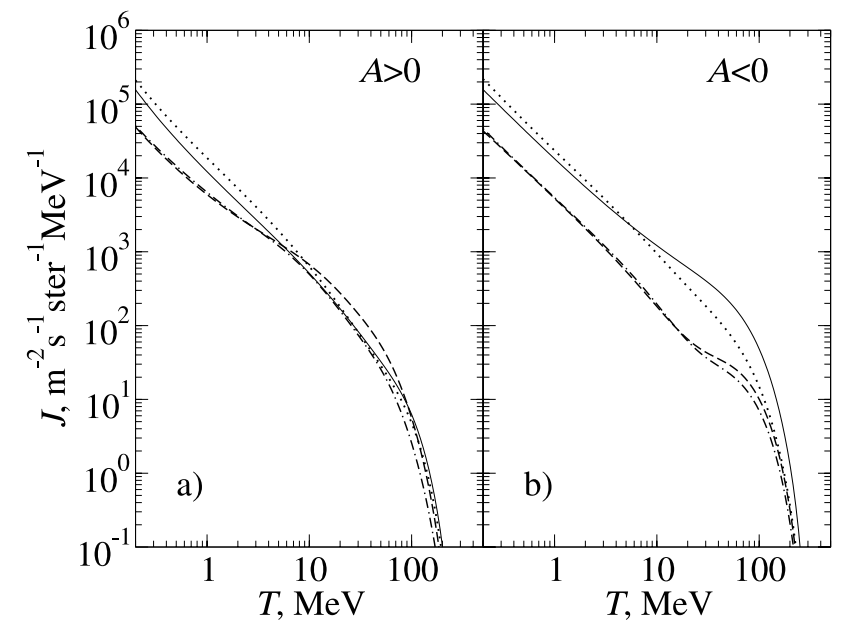

FIG. 7.-(a) ACR spectra at $r=r_{\mathrm{sh}}$ in the ecliptic (solid line), pole (dashed line), and at $r=120 \mathrm{AU}$ in the downstream region in the ecliptic (dotted line) and pole (dot-dashed line) for the positive solar cycle. Note compressional reacceleration below $10 \mathrm{MeV}$ at low latitudes. (b) Same for the negative solar cycle.

Conversely, during the negative cycle, particles drift toward the ecliptic, and their intensity at high latitudes remains low as seen in Figure 6. In the latter case the maximum is in the ecliptic at the TS location.

In addition, Figure 5 shows that the shock is elongated in the poleward direction as a result of solar wind dynamic pressure variation with heliolatitude. Note that in global heliospheric models the TS tends to be elongated in the direction parallel to the interstellar flow even for a fully isotropic solar wind, owing to an increased thermal pressure in the direction of the nose of the heliopause. It is, however, not possible to capture this effect in a two-dimensional polar model. A comparative study of isotropic versus anisotropic solar wind models can be found in Pauls \& Zank (1997), who also included interstellar hydrogen in their simulations.

Figure 7 compares ACR differential intensities $J=f p^{2}$ at the TS and in the heliosheath at different latitudes. Because the injection rate is higher in the ecliptic, owing to greater PUI densities, low-energy intensities are higher in the ecliptic than at the pole by a factor of 3-4.5. Lower energy portions of the spectra can be approximated by power laws with indexes varying between -1.25 and -1.35 , consistent with a relatively weak shock with a compression ratio of about 3 (see $\S 5$ ). Several spectra display a characteristic bump before the cutoff energy. This feature is produced by a combination of drift effects, spherical geometry effects, and modified shock effects (Florinski \& Jokipii 2003). Because the spectra differ so much between the $A>0$ and $A<0$ cycles, we can conclude that the first of these effects dominates the other two.

The $A>0$ case shows strong drift effects, where the intensity just below the rollover at high latitude exceeds the intensity at low latitude, despite the difference in injection rates. This situation corresponds to the maxima in Figure 5. Because drifts act in the opposite direction during the $A<0$ part of the solar cycle (i.e., $\boldsymbol{v}_{d}$ at the TS is directed toward the ecliptic plane), the polar intensity is lower at high latitudes than at low latitudes. This effect is particularly noticeable at $\sim 50 \mathrm{MeV}$, where equatorial fluxes exceed the polar fluxes by a factor of 10 .

A noteworthy feature of ACR spectra downstream of the TS is enhanced intensity at energies below $10 \mathrm{MeV}$ in the ecliptic region. This effect is clearly seen in both panes of Figure 7 and has a magnitude of $40 \%-50 \%$. This reacceleration of ACRs in the heliosheath is a direct consequence of the slowdown of the shocked solar wind by charge exchange with interstellar hydrogen. The magnitude of this effect can be estimated by noting that downstream of the shock the approximate condition of incompressibility of the flow is (Khabibrakhmanov et al. 1996)

$$
\gamma \nabla \cdot \boldsymbol{u}=-\nu / 2
$$

where $\nu \simeq 10^{-8} \mathrm{~s}^{-1}$ is the rate of charge exchange. A spherically symmetric solution to equation (25) is

$$
u=u_{s}\left(\frac{r_{s}}{r}\right)^{2}-\frac{\nu r_{s}}{6 \gamma}\left(\frac{r}{r_{s}}-\frac{r_{s}^{2}}{r^{2}}\right)
$$

( $u_{s}$ being the speed just downstream of the TS) showing a faster wind speed decrease than the usual $r^{-2}$. Because ACRs in the heliosheath are strongly coupled to the plasma, the acceleration timescale will be on the order of $\nu^{-1}$. Examination of equation (13) together with (25) shows that the timescale for acceleration $\tau_{\text {acc }}=6 \gamma / \nu \sigma \simeq 8 \mathrm{yr}$ is larger that the convective timescale $\tau_{\text {conv }}=\left(r_{b}-r_{s}\right) / u \simeq 5 \mathrm{yr}$, where $r_{b} \simeq 150 \mathrm{AU}$ is the distance to the external boundary (heliopause). We therefore expect that compressive reacceleration is less efficient than shock acceleration, which proceeds at a significantly higher rate at low energies, but is limited by upstream cooling and particle escape near the cutoff. It should be kept in mind that the above estimate of $\tau_{\text {conv }}$ is appropriate for a spherically symmetric case and ignores such effects as flow turning on approach to the heliopause and stagnation on the symmetry axis. The actual low-energy ACR storage time in the heliosphere could be significantly longer, allowing for more compression.

Note that the compression effect is absent at high latitudes and at high particle energies. This happens because of a significantly longer diffusion lengths (diffusion coefficient increases with increasing latitude and energy), facilitating particle escape across the heliopause, and lesser degree of downstream flow mediation by PUI in polar regions, owing to smaller charge exchange rate (see $\S 5$ ). The influence of diffusion on downstream acceleration is discussed in some detail in the Appendix. Spectra shown in Figure 7 should be viewed in the context of Figure 11 of the Appendix, which demonstrates that higher energy particles experience less compressional acceleration that lower energy ones. Figure 11 shows that the amount of acceleration decreases already for modest values of the downstream diffusion coefficient $\kappa_{r r}>0.05-0.1 u r_{s}$. Because particles at these energies are still accelerated at the TS, shock and post-shock spectra cross over at energies below the coolinginduced cutoff, as seen in Figure 7. Note that the crossover occurs at lower energies in Figure $7 b$ than in Figure $7 a$. This can be understood by noting that inward drift along the neutral sheet during periods when the magnetic polarity is negative effectively reduces the total outward velocity, leading to faster diffusive escape across the HP (see the Appendix for a more rigorous explanation).

To validate our model against ACR observations we compare model-calculated spectra with Voyager 1 and 2 measurements during the period of 1998-1999.5, when Voyager 1 was $\sim 70 \mathrm{AU}$ and Voyager 2 was $\sim 55 \mathrm{AU}$ away from the Sun. This comparison is shown in Figure 8. As can be seen, the current model shows a reasonable degree of agreement with the data. Spectra at the TS are also quite similar to those calculated by Cummings et al. (2002) for their weak shock case. The modelcalculated radial gradient between 70 and $55 \mathrm{AU}$ is larger than 


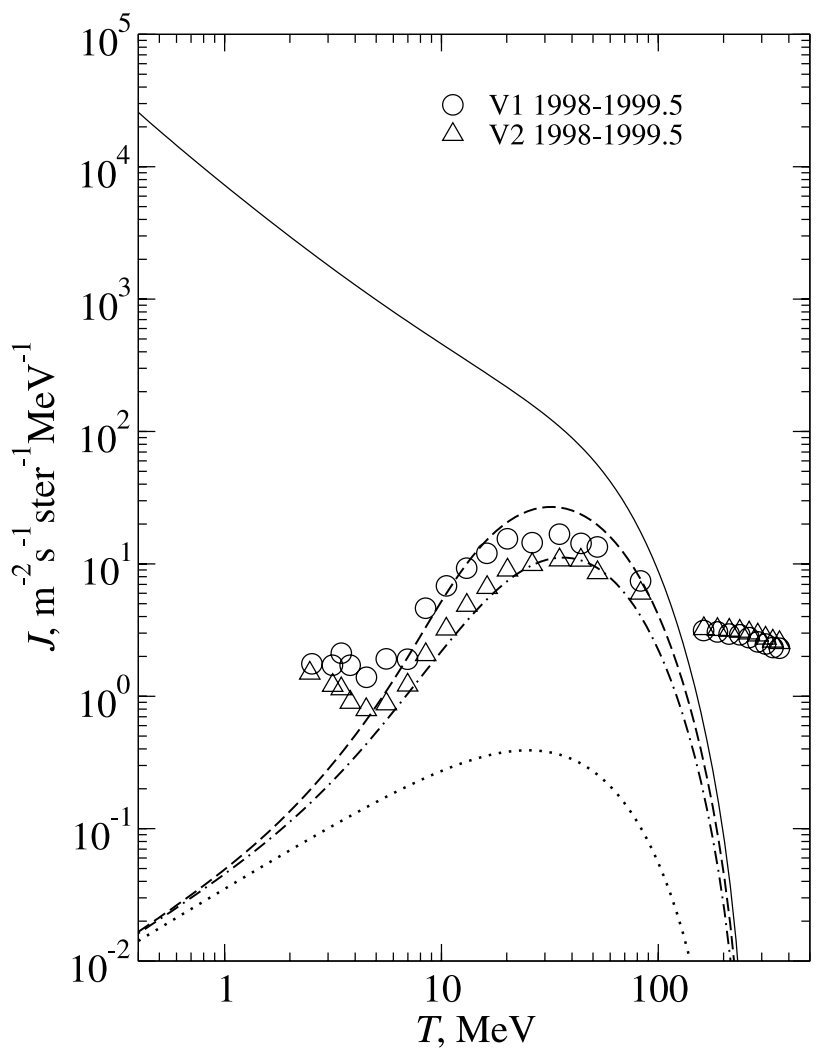

FIG. 8.-ACR proton spectra for the positive solar cycle at $30^{\circ}$ latitude. Solid line is the spectrum at the shock $r_{s}=94 \mathrm{AU}$, dashed line at $70 \mathrm{AU}$, dotdashed line at $55 \mathrm{AU}$, and dotted line at $4 \mathrm{AU}$. Voyager 1 and 2 spectra (Cummings et al. 2002) are shown for comparison for the time period 1998 1999.5, during which the average Voyager 1 distance was $72 \mathrm{AU}$ and latitude $33^{\circ}$, and Voyager 2 was at $56 \mathrm{AU}$ and $-19^{\circ}$, respectively.

that observed by about a factor of 1.5-2. Because Voyager 2 was at a latitude lower than $30^{\circ}$, the computed gradient between the two spacecraft would be even larger. In their model Cummings et al. (2002) overcame the large gradient problem by using a diffusion coefficient that was sufficiently large at distances corresponding to the Voyager locations resulting in small gradients, but decreasing in the immediate vicinity of the shock, presumably as a result of shock-generated turbulence, thus ensuring that the maximum energy attainable by ACRs is consistent with the predicted location of the spectral rollover. Note also that because Voyager 1 was in the northern hemisphere and Voyager 2 in the southern hemisphere, actual gradients at both spacecraft locations may be different. Further improvements to our self-consistent diffusion model will be needed to include latitudinal dependence of the turbulence parameters (amplitude and correlation length), as well as possible additional turbulence production by cosmic-ray driven instabilities inside the shock precursor (e.g., Drury \& Falle 1986). Despite this, the present model allows us for the first time to calculate ACR effects on the heliosphere and the TS in a way that is reasonably consistent with measured ACR intensities. These effects are discussed in the next section.

\section{ACR DYNAMIC EFFECTS AND SHOCK MODIFICATION}

Figure 9 shows the radial dependence of the solar wind flow parameters. The $A>0$ self-consistent case is shown. The

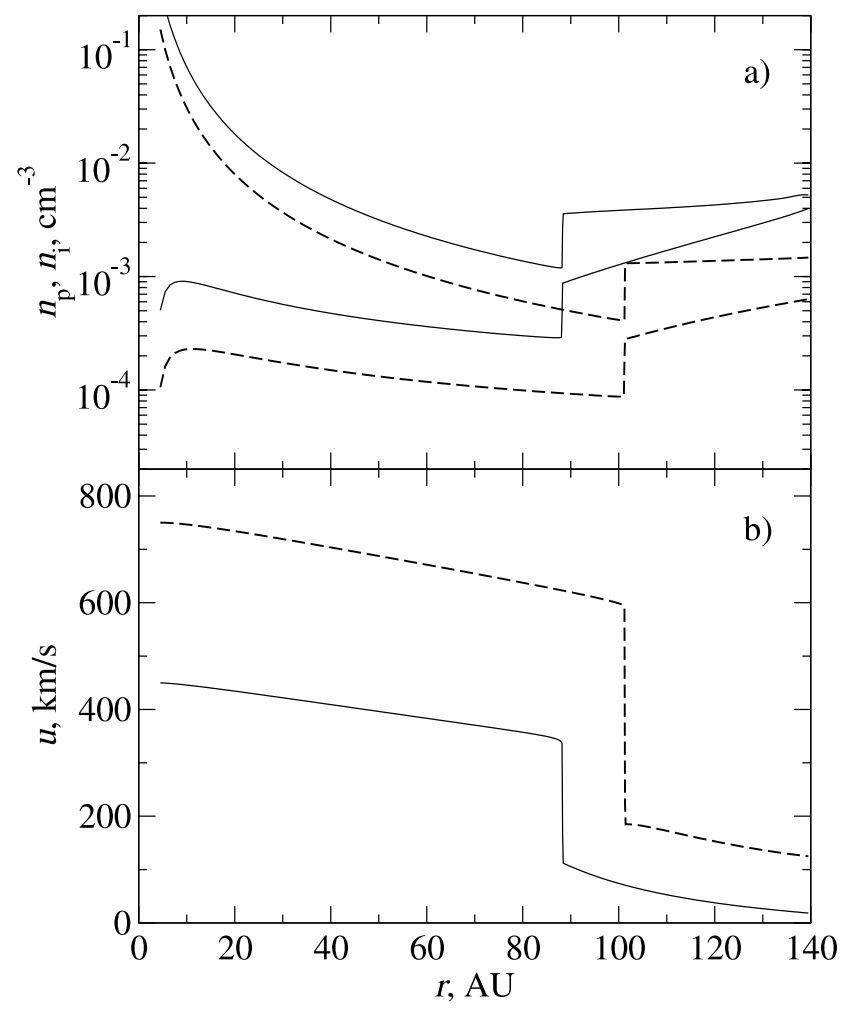

FIG. 9.- Solar wind flow parameters from the self-consistent model $(A>0$ case). (a) Plasma (top two curves) and PUI (bottom two curves) density at $0^{\circ}$ heliographic latitude (solid lines) and at $90^{\circ}$ (dashed lines). (b) Solar wind radial flow velocity in the ecliptic (solid line) and at $90^{\circ}$ (dashed line).

$A<0$ case is very similar when viewed on a large spatial scale. Because the PUI density increases rapidly beyond the shock, the flow at the external boundary consists mostly of PUIs. From equation (24) for an incompressible flow, the PUI density increases as

$$
n_{i}=n_{\mathrm{is}}+\frac{\nu n_{p} r_{s}}{3 u_{s}}\left(\frac{r^{3}}{r_{s}^{3}}-1\right)
$$

where $n_{\text {is }}$ is the PUI density immediately downstream of the shock, but the growth will be even faster in view of equation (26). Figure $9 b$ shows the solar wind deceleration by charge exchange momentum removal. The velocity decrease upstream of the TS is essentially linear in agreement with Lee (1997). The amount of deceleration is $\sim 14 \%$ between 1 and $60 \mathrm{AU}$ in the ecliptic, which is consistent with the Voyager 2 plasma detector data (Wang \& Richardson 2003). Because the combined solar wind and PUI sound speed upstream of the shock is much larger than that of the solar wind itself, the total Mach number is reduced significantly and the TS is relatively weak (Isenberg 1997), with a compression ratio of about $s=3.25$ at high latitudes and $s=3.05$ in the ecliptic plane, before ACR dynamic effects are taken into account.

Figure 10 compares ACR dynamical effects on the solar wind plasma for the positive and negative solar cycles. One can see that this effect is quite small, owing to the relatively small ACR pressure attainable at the shock. The maximum $p_{c}$ in the ecliptic plane is found to be $0.07 \mathrm{eV} \mathrm{cm}^{-3}$ for $A>0$ and $0.16 \mathrm{eV} \mathrm{cm}^{-3}$ for $A<0$, significantly less than the solar 


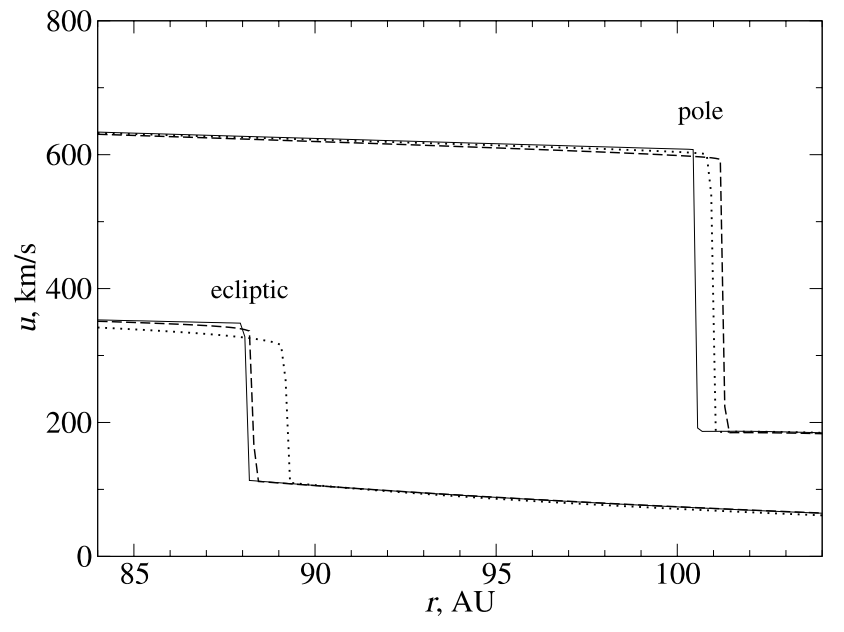

FIG. 10.- Solar wind velocity near the TS at $0^{\circ}$ and $90^{\circ}$. Solid lines show the shock location without cosmic-ray dynamic effects, dashed lines show the $A>0$ self-consistent case, and dotted lines show the $A<0$ case.

wind dynamic pressure upstream or plasma thermal pressure downstream of the shock, both of which are $\sim 1 \mathrm{eV} \mathrm{cm}^{-3}$. ACR pressure gradients cause the shock to move to a larger heliocentric distance by less than $1 \mathrm{AU}$. The $A<0$ case shows a stronger precursor in the ecliptic region owing to a higher ACR pressure at the shock. Note that most of the excess pressure is carried by particles with intermediate to high energies (i.e., the bump region; compare Figs. $7 a$ and $7 b$ ). The length of the precursor during the negative portion of the solar cycle is increased because of smaller ACR intensity radial gradient in this case. The smaller gradient is a combined effect of increased average diffusion coefficient (ACRs are, on average, more energetic) and inward drifts in the ecliptic plane.

Our results show that the gas subshock compression ratio is decreased by $0.1-0.2$, depending on the solar cycle and the latitude. The weakest shock is observed at low latitudes during the negative cycle with a compression ratio of 2.9. Clearly, ACR shock modification is secondary compared to that of charge exchange, which reduces the compression ratio from $s=4.0$ to 3.05 in the ecliptic.

Finally, we would like to estimate whether using different shock parameters is likely to change the result that the TS is only weakly mediated by ACRs. To do this, we integrate the published shock spectra at $30^{\circ}$, calculated by Cummings et al. (2002) for the positive solar cycle, to find the total cosmic-ray pressure at the shock. Because the published spectra are for $60^{\circ}$, we compare them with our results for the same latitude, keeping in mind that the pressure in the ecliptic is $\sim 50 \%$ higher. We also include the energy density of anomalous helium, which we estimate to be approximately $10 \%$ that of anomalous hydrogen. For their weak shock case (compression ratio 2.4), the published spectra give $p_{c, \mathrm{H}}=0.075 \mathrm{eV} \mathrm{cm}^{-3}$ and $p_{c, \mathrm{He}}=0.0095 \mathrm{eV} \mathrm{cm}^{-3}$ for the common energy interval from $200 \mathrm{keV}$ to infinity, while in the strong shock case (not a very realistic scenario) the pressures are $p_{c, \mathrm{H}}=0.023 \mathrm{eV} \mathrm{cm}^{-3}$ and $p_{c, \mathrm{He}}=0.0023 \mathrm{eV} \mathrm{cm}^{-3}$. The value we calculated for the same latitude falls in between with $p_{c, \mathrm{H}}=0.048 \mathrm{eV} \mathrm{cm}^{-3}$ (subshock compression ratio $s=3.1$ ). This estimate shows that the total ACR pressure can be up to $80 \%$ larger at the shock, which is still relatively small. We expect that in the most extreme scenario the subshock compression ratio will be reduced by 0.3 to $s=2.8$ at low latitude.

\section{DISCUSSION}

The model introduced here is a significant improvement over the GCR model described in Florinski \& Jokipii (1999) and is an important step in the direction of building a fully self-consistent global model of the heliosphere. By using an adaptive mesh we are able to properly study the acceleration and propagation of the anomalous cosmic rays with small diffusive length scales and their effects on the solar wind. Our main finding is that the ACRs with energies above $100 \mathrm{keV}$ have only a small dynamical effect on the solar wind flow because their pressure is small compared to the solar wind dynamic pressure, as constrained by the spectra observed by Voyager. This result is consistent with that of Fahr et al. (2000) obtained using a hydrodynamic cosmic-ray model.

We have not discussed the possibility of an enhanced injection rate. If particles are accelerated diffusively, the subshock compression ratio decreases significantly, and the ACR spectra attain a concave shape owing to the fact that lower energy particles are unable to traverse the entire shock structure (le Roux \& Fichtner 1997). The reason we do not study this case is because high injection rates result in very steep spectra at small momenta, where most of the ACR energy will reside. This necessitates including particles at even lower energies than those considered here (i.e., below $100 \mathrm{keV}$ ), which would impose a severe constraint on the resolution of the numerical scheme. In addition, particles with $\mathrm{keV}$ energies cannot be adequately described by the diffusion theory, as shown in $\S 3$. It can be shown that if the spectra extends below $100 \mathrm{keV}$ with the same power law as prescribed by the shock compression ratio (i.e., -1.25 for a shock with a strength of 3.0), particles below $100 \mathrm{keV}$ contribute no more than $0.03 \mathrm{eV} \mathrm{cm}^{-3}$ to $p_{c}$. Because the actual spectrum can be either softer (diffusive acceleration at a modified shock) or harder (MRI acceleration), it is difficult to estimate theoretically just how much of the total ACR pressure is missing. From an observational perspective, recent results from the HENA instrument on board the IMAGE spacecraft place an upper limit on $10-100 \mathrm{keV}$ ion flux at the TS that appears to exclude the possibility of enhanced intensities in this energy interval (E. C. Roelof 2004, in preparation).

Particles with $\mathrm{keV}$ energies will be strongly coupled to the background plasma and would produce a shock precursor on sub-AU scales. A study of this phenomenon requires a different model operating on much smaller scales. The model of le Roux et al. (2000) showed that the shock structure can be significantly modified on scales $\ll 0.1$ AU owing to pressure exerted by MRI-accelerated ions. However, such modification is effectively invisible on the scales considered here, and it does not affect diffusive acceleration of ACRs. A more complete model must be able, in general, to take into account particle anisotropy and include turbulence generation near the shock produced by hydrodynamic instabilities in the presence of cosmic-ray pressure gradients (Drury \& Falle 1986; Zank et al. 1990). The latter would tend to reduce the ACR diffusive length just upstream of the shock and modify injection into the diffusive shock acceleration process.

Similar difficulties arise when attempting to model the heliosphere during periods close to solar maxima. Because the solar wind is more turbulent during those periods, diffusion is suppressed and drifts are believed to be essentially inoperative. Voyager observations show that the radial diffusion mean free path is smaller by a factor of 10-15 compared to solar minima, at least for $\sim 1 \mathrm{GeV}$ particles (Cummings \& Stone 2003; Fujii et al. 2003). Given the reduction in drifts, the 
diffusion approximation criterion (19) can be more readily satisfied and the Parker model extendable to even lower proton energies. To model these conditions, an increase in resolution by a factor over 10 is required, equivalent to adding three to four extra levels of refinement. Clearly, suppressed diffusion would lead to ACRs being accelerated to larger energies as the spectral cutoff condition $\left(u r_{s} / \kappa_{r r} \leq\right.$ number of order 1) is more difficult to satisfy (Potgieter \& Langner 2003). What is not clear is whether ACR pressure during solar maximum will significantly exceed its solar minimum value because, while shock and stochastic pre-acceleration will proceed faster owing to more numerous propagating shocks and enhanced turbulence levels, acceleration at the TS can be inhibited by large excursions of the shock itself. The situation is further complicated by the presence of global merged interaction regions, viewed as propagating diffusion barriers, that introduce temporal variability in cosmic-ray intensity (see, e.g., Potgieter et al. 2001 for a review).

As mentioned earlier, the current AMR model applies to the region of the heliosphere that is facing the interstellar wind, extending from the inner solar system to a distance smaller than the distance to the heliopause, lest the flow stagnation effects become important. Nevertheless, we should mention possible effects of the more complex heliosheath geometry on ACR propagation. One particular feature of the diffusion tensor in this region is the so-called modulation wall, discussed in Florinski et al. (2003). The wall is quite impervious to incoming low-energy GCRs and will hinder ACR escape into the LISM. At the same time, the fact that the magnetic field is tightly wound in the heliosheath would make ACR return to the TS unlikely as well. Generally, ACRs will be convected along the heliopause, gradually diffusing across its surface and escaping into the LISM.

The present model should be sufficiently accurate in predicting ACR intensities near the TS in both up- and downwind directions and in the inner heliosphere. The TS is elongated in the downwind direction, with a stand-off distance between 120 and $180 \mathrm{AU}$ on the symmetry axis, depending on the magnitude of the interstellar magnetic field (Florinski et al. 2003, 2004), and the scales will have to be adjusted accordingly. The distance to the shock, however, is not so much of a factor controlling the properties of the spectra, and we expect that the latter will not be qualitatively different from the spectra discussed here. As shown in Florinski \& Jokipii (2003), spherical shock spectral features (i.e., the cutoff and bump) depend almost exclusively on the upstream shock parameter $\eta=u r_{s} / \kappa_{r r}$, which is weakly dependent on $r_{s}$, since $\kappa_{r r} \sim r^{1+a}$, where $a \ll$ 1 , according to mean free path estimates based on Voyager gradients (Cummings \& Stone 2003; Fujii et al. 2003). Neutral hydrogen density is smaller by about a factor of 2 in the downwind direction as a result of neutral particle deflection away from the symmetry axis. However, because the shock is located farther away from the Sun, the wind is decelerated by about the same amount by the time it reaches the TS and the shock compression ratio is approximately the same up- and downwind (Fahr et al. 2000; Florinski et al. 2003). Quantitatively, ACR intensities will likely be different in the two directions because the density of PUIs is smaller by about a factor of 2 downwind (Fahr et al. 2000), leading to ACR intensity being smaller by the same factor. Most PUIs are produced within $10 \mathrm{AU}$ from the Sun; however, their density falls off $\sim r^{-1}$, i.e., slower than the wind dynamic pressure, at large heliocentric distances. Consequently, ACR TS modification may be similar in the direction of the heliotail to that upwind. Finally, at larger distances in the heliosheath and heliotail we might expect ACRs to be energized additionally by converging plasma flows resulting from charge exchange (Czechowski et al. 2001; Izmodenov \& Alexashov 2003; Florinski et al. 2003).

The principal results of this paper can be summarized as follows:

1. An adaptive mesh numerical code is used to investigate self-consistently the interaction between the solar wind, interstellar neutrals, and ACR hydrogen. The code is capable of efficiently solving the transport equation with a good resolution without an excessive increase in computational time. Test results are consistent with "traditional" modulation models, and all characteristic features of the particle distribution are captured.

2. Solar wind slowdown by charge exchange with interstellar atoms produces convergent flows near the ecliptic plane in the heliosheath where some ACRs are accelerated beyond their shock-attained energies. The extra amount of acceleration is on the order of $50 \%$ and decreases with increasing energy, owing to diffusive losses across the heliopause. This effect is visible at energies below $10 \mathrm{MeV}$ and is stronger during the positive solar cycles. It is absent in traditional modulation models that ignore neutral atoms.

3. ACRs have a relatively small effect on the location and compression ratio of the solar wind TS during the periods near solar minima. In our model, the subshock moves away from the Sun by about $1 \mathrm{AU}$, and its compression ratio is decreased by only about $5 \%$. At low latitudes, this effect is more important during the $A<0$ solar cycle than for the $A>0$ magnetic field polarity.

V. F. and G. P. Z. acknowledge support by NASA grants NAG5-11621 and NAG5-12903. The work of J. R. J. was supported, in part, by NASA grants NAG5-10893 and NAG510990 and by the NSF under grant ATM-0330829. The work of E. C. S. and A. C. C. was supported by NASA under contract NAS7-03001.

\section{APPENDIX A}

\section{COSMIC-RAY ACCELERATION DOWNSTREAM OF THE TERMINATION SHOCK}

Here we solve the ACR transport equation (13) in the presence of a decelerated flow downstream of the TS under the assumption of spherical symmetry. The steady-state transport equation

$$
u \frac{\partial f}{\partial r}-\frac{1}{r^{2}} \frac{\partial}{\partial r}\left(\kappa_{r r} r^{2} \frac{\partial f}{\partial r}\right)=\frac{\nabla \cdot u}{3} \frac{\partial f}{\partial \ln p}
$$


can be further simplified by assuming that the diffusion coefficient $\kappa_{r r}=\kappa$ is a constant in phase space and that, consequently, the distribution function retains its power-law spectral dependence $f \sim p^{-\sigma}$ throughout the heliosheath region. Using equations (25) and (26) the transport equation (A1) is reduced to

$$
\frac{d^{2} f}{d x^{2}}-\left(\frac{\eta+\eta_{c}}{x^{2}}-\frac{2}{x}-\eta_{c} x\right) \frac{d f}{d x}+\sigma \eta_{c} f=0
$$

where $x=r / r_{s}, \eta=u_{s} r_{s} / \kappa$ is the ordinary downstream shock parameter, and $\eta_{c}=\nu r_{s}^{2} /(6 \gamma \kappa)$ is the second shock parameter associated with charge exchange. The boundary conditions that accompany the above equation are $f(1)=f_{s}$ and $f(b)=0$, where $b=r_{b} / r_{s}$.

To solve equation (A2) approximately, we expand $f$ in powers of $\eta_{c}$, so that $f \simeq f_{0}+\eta_{c} f_{1}$. The zeroth-order equation

$$
\frac{d^{2} f_{0}}{d x^{2}}-\left(\frac{\eta}{x^{2}}-\frac{2}{x}\right) \frac{d f_{0}}{d x}=0
$$

clearly has the following solution

$$
f_{0}=f_{s} \frac{e^{-\eta / b}-e^{-\eta / x}}{e^{-\eta / b}-e^{-\eta}}
$$

The function $f_{1}$ satisfies the equation

$$
\frac{d^{2} f_{1}}{d x^{2}}-\left(\frac{\eta}{x^{2}}-\frac{2}{x}\right) \frac{d f_{1}}{d x}=-\frac{f_{s}}{e^{-\eta / b}-e^{-\eta}}\left[\frac{\eta e^{-\eta / x}}{x^{2}}\left(\frac{1}{x^{2}}-x\right)+\sigma\left(e^{-\eta / b}-e^{-\eta / x}\right)\right] .
$$

Equation (A5) is solved by means of a Green's function. The exact solution is somewhat cumbersome; however, it can be simplified substantially by assuming that the diffusion coefficient downstream of the shock is not very large, i.e., $\eta \gg 1$. Then we can use the following approximate expressions for the integrals appearing in the solution:

$$
\int x e^{-\eta / x} d x \simeq \frac{x^{3}}{\eta} e^{-\eta / x}, \quad \int x^{2} e^{-\eta / x} d x \simeq \frac{x^{4}}{\eta} e^{-\eta / x},
$$

which follow from the asymptotic representation of the exponential integral for large arguments (Gautschi \& Cahill 1965). After some algebraic manipulations we obtain

$$
\begin{aligned}
f_{1}= & \frac{f_{s}}{\left(e^{-\eta / b}-e^{-\eta}\right)^{2}}\left[\left(\frac{x^{3}+2}{2 x}-\frac{x^{3}-1}{\eta}\right) e^{-\eta / x}\left(e^{-\eta / b}-e^{-\eta}\right)-\left(\frac{b^{3}+2}{2 b}-\frac{b^{3}-1}{\eta}\right) e^{-\eta / b}\left(e^{-\eta / x}-e^{-\eta}\right)\right. \\
& -\frac{3}{2} e^{-\eta}\left(e^{-\eta / b}-e^{-\eta / x}\right)+\frac{\sigma\left(x^{4}-1\right)}{\eta^{2}}\left(e^{-\eta / b}-e^{-\eta}\right)\left(e^{-\eta / b}-e^{-\eta / x}\right)+\frac{\sigma x^{3}}{3 \eta}\left(e^{-\eta / b}+e^{-\eta / x}\right)\left(e^{-\eta / b}-e^{-\eta}\right) \\
& \left.-\frac{2 \sigma b^{3}}{3 \eta} e^{-\eta / b}\left(e^{-\eta / x}-e^{-\eta}\right)-\frac{\sigma}{3 \eta}\left(e^{-\eta / b}-e^{-\eta / x}\right)\left(e^{-\eta / b}+e^{-\eta}\right)\right] .
\end{aligned}
$$

Figure 11 compares the exact solution to equation (A2), obtained by numerical integration, with the approximate solution (A7) using typical values $u_{s}=100 \mathrm{~km} \mathrm{~s}^{-1}, b=1.5, \sigma=5$, and $\alpha^{-1}=\eta / \eta_{c}=6$. The first-order solution is very similar to the exact solution when $\eta$ is not very large and loses accuracy in the limit of small diffusion. The maximum amount of compressional acceleration can be obtained in the zero diffusion limit $\eta, \eta_{c} \rightarrow \infty$. In that case, the solution to equation (A2) is

$$
f(\eta \rightarrow \infty)=f_{s}\left[1-\alpha\left(x^{3}-1\right)\right]^{-\sigma / 3}
$$

(this solution obviously does not satisfy the right boundary condition). Using the above downstream parameters, the maximum amount of compression in the heliosheath for the lowest energy ACRs is estimated to be 2.3.

An improved approximate solution to equation (A2) can be obtained by considering a boundary layer expansion near $x=b$ in the small diffusion limit. We only show the zeroth-order solution here. The first or higher order solutions can be obtained using the standard boundary layer perturbation method by rewriting equation (A2) in terms of the inner variable $\xi=\eta(b-x)$, expanding the resulting expression in powers of $\eta^{-1}$ and matching the inner and the outer solutions. The zeroth-order diffusion-corrected expression is readily shown to be 


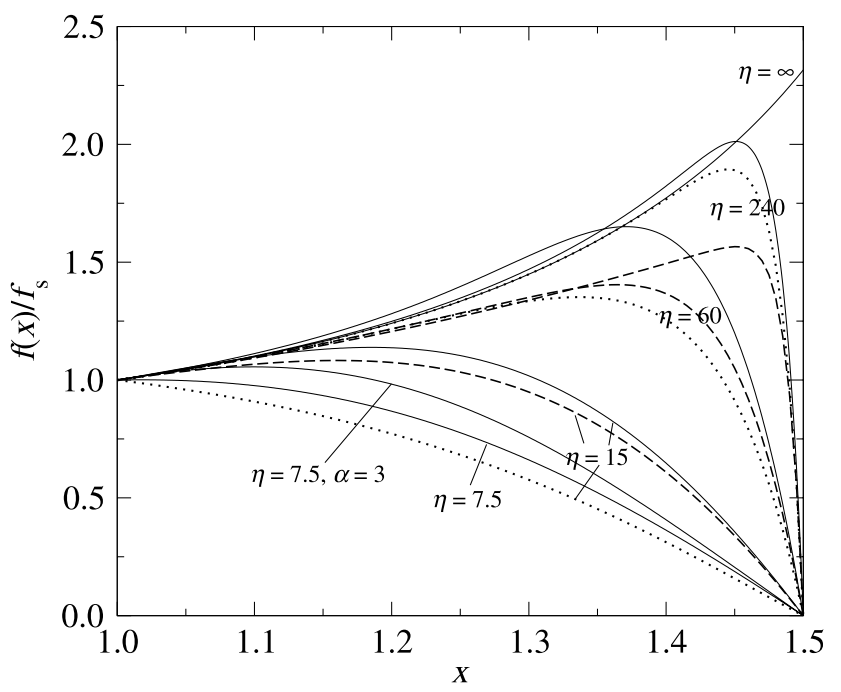

Fig. 11.-Numerical (solid lines) and approximate analytic solutions to eq. (A2) for $\eta=15,60,240$, and $\infty$ and $\alpha^{-1}=6$. Dashed lines correspond to the approximation (A7), dotted lines correspond to the approximation (A9). In addition, a numerical solution for a relatively large diffusion coefficient $(\eta=7.5)$ shows no acceleration at all, and a solution with $\eta=7.5, \alpha^{-1}=3$ demonstrates the effect of a sunward drift velocity during the $A<0$ periods (see text).

$$
f \simeq f_{s}\left[1-\alpha\left(x^{3}-1\right)\right]^{-\sigma / 3}-f_{s} \frac{e^{-\alpha b \eta(b-x)}-e^{-\alpha b \eta(b-1)}}{1-e^{-\alpha b \eta(b-1)}}\left[1-\alpha\left(b^{3}-1\right)\right]^{-\sigma / 3}
$$

This solution is also plotted in Figure 11. As expected, the accuracy of this approximation is high in the limit of the small diffusion and degrades with decreasing $\eta$.

Figure 11 demonstrates that less energetic particles $(\eta>60)$ experience more acceleration. At larger energies escape across the HP dominates acceleration (see the $\eta=7.5$ curve, which shows no intensity enhancement). Note that sunward drifts during the $A<0$ solar cycle phase will further inhibit acceleration. Indeed, an inward drift can be viewed as an effective reduction in $u$ and, hence, an increase in the diffusive length leading to a faster rate of escape. This effect is best seen by comparing the solid curves for $\eta=15, \alpha^{-1}=6$ and $\eta=7.5, \alpha^{-1}=3$ in Figure 11. The two curves correspond to the same $\eta_{c}$, but the effective velocity $u$ is smaller by a factor of 2 as a result of drifts in the latter case. Clearly, the latter case shows less acceleration. Similarly, outward drifts during the $A>0$ solar minima will have an opposite effect; i.e., acceleration will be enhanced.

Axford, W. I. 1972, in Solar Wind, ed. C. P. Sonnett et al. (Washington, DC: NASA), 609

Banaszkiewicz, M., \& Ziemkiewicz J. 1997, A\&A, 327, 392

Berger, M. J., \& Colella, P. 1989, J. Comput. Phys., 82, 64

Bieber, J. W., Wanner, W., \& Matthaeus, W. H. 1996, J. Geophys. Res., 101,2511

Chalov, S. V., \& Fahr, H. J. 1996, Sol. Phys., 168, 389 1997, A\&A, 326, 860

Chalov, S. V., Fahr, H. R., \& Izmodenov, V. V. 1997, A\&A, 320, 659

Christian, E. R., Cummings, A. C., \& Stone, E. C. 1988, ApJ, 334, L77 1995, ApJ, 446, L105

Cummings, A. C., \& Stone, E. C. 1998, Space Sci. Rev., 83, 51

2003, in Proc. 28 Int. Cosmic Ray Conf. (Tokyo), 2897

Cummings, A. C., Stone, E. C., \& Steenberg, C. D. 2002, ApJ, 578, 194

Czechowski, A., Fichtner, H., Grzedzielski, S., Hilchenbach, M., Hsieh, K. C., Jokipii, J. R., Kausch, T., Kota, J., \& Shaw, A. 2001, A\&A, 368, 622

Donohue, D. J., \& Zank, G. P. 1993, J. Geophys. Res., 98, 19005

Drury, L. O'C., \& Falle, S. A. E. G. 1986, MNRAS, 223, 353

Ellison, D. C., Jones, F. C., \& Baring, M. G. 1999, ApJ, 512, 403

Fahr, H. J., Kausch, T., \& Scherer, H. 2000, A\&A, 357, 268

Fichtner, H. 2001, Space Sci. Rev., 95, 639

Fisk, L. A., Forman, M. A., \& Axford, W. I. 1973, J. Geophys. Res., 78, 995

Florinski, V. 2001, Ph.D. thesis, Univ. Arizona

Florinski, V., \& Jokipii, J. R. 1999, ApJ, 523, L185 2003, ApJ, 591, 454

Florinski, V., Pogorelov, N. V., Zank, G. P., Wood, B. E., \& Cox, D. P. 2004, ApJ, 604, 700

Florinski, V., Zank, G. P., \& Pogorelov, N. V. 2003, J. Geophys. Res., 108,1228

Fujii, Z., McDonald, F. B., \& Moraal, H. 2003, Adv. Space Res., 32, 639

Gautschi, W., \& Cahill, W. F. 1965, in Handbook of Mathematical Functions, ed. M. Abramowitz \& I. A. Stegan (New York: Dover), 227

\section{EFERENCES}

Giacalone, J., \& Jokipii, J. R. 1999, ApJ, 520, 204

Giacalone, J., Jokipii, J. R., Decker, R. B., Krimigis, S. M., Scholer, M., \& Kucharek, H. 1997, ApJ, 486, 471

Gloeckler G., Fisk, L. A., \& Geiss, J. 1997, Nature, 386, 374

Goldstein, B. E., Neugebauer, M., Phillips, J. L., Bame, S., Gosling, J. T.,

McComas, D., \& Wang, Y.-M. 1996, A\&A, 316, 296

Isenberg, P. A. 1986, J. Geophys. Res., 91, 9965 1997, Geophys. Res. Lett., 24, 623

Izmodenov, V., \& Alexashov, D. 2003, Astron. Lett., 29, 58

Jokipii, J. R. 1971, Rev. Geophys. Space Phys., 9, 27

. 1982, ApJ, 255, 716

—. 1986, J. Geophys. Res., 91, 2929

- 1987, ApJ, 313, 842

Jokipii, J. R., \& Giacalone, J. 1998, Space Sci. Rev., 83, 123

Jokipii, J. R., \& Kota, J. 1989, Geophys. Res. Lett., 16, 1

Jokipii, J. R., Kota, J., \& Merenyi, E. 1993, ApJ, 405, 782

Kang, H., Jones, T. W., LeVeque, R. J., \& Shyue, K. M. 2001, ApJ, 550, 737 Khabibrakhmanov, I. K., Summers, D., Zank, G. P., \& Pauls, H. L. 1996, J. Geophys. Res., 101, 20003

Ko, C. M., Jokipii, J. R., \& Webb, G. M. 1988, ApJ, 326, 761

Ko, C. M., \& Webb, G. M. 1988, ApJ, 325, 296

Lee, M. A. 1997, in Cosmic Winds and the Heliosphere, ed. J. R. Jokipii et al. (Tucson: Univ. Arizona Press), 875

Lee, M. A., \& Axford, W. I. 1988, A\&A, 194, 297

Lee, M. A., Shapiro, V. D., \& Sagdeev, R. Z. 1996, J. Geophys. Res., 101, 4777 le Roux, J. A., \& Fichtner, H. 1997, J. Geophys. Res., 102, 17365

le Roux, J. A., Zank, G. P., Fichtner, H., \& Ptuskin, V. S. 2000, Geophys. Res. Lett., 27, 2873

le Roux, J. A., Zank, G. P., \& Ptuskin, V. S. 1999, J. Geophys. Res., 104, 24845 McNutt Jr., R. L., Lyon, J., \& Goodrich, C. C. 1998, J. Geophys. Res., 103, 1905

Parker, E. N. 1965, Planet. Space Sci., 13, 9 
Pauls, H. L., \& Zank, G. P. 1997, J. Geophys. Res., 102, 19779

Pesses, M. E., Jokipii, J. R., \& Eichler, D. 1981, ApJ, 246, L85

Potgieter, M. S., Burger, R. A., \& Ferreira, S. E. S. 2001, Space Sci. Rev., 97, 295

Potgieter, M. S., \& Langner, U. W. 2003, Adv. Space Res., 32, 687

Potgieter, M. S., \& Moraal, H. 1985, ApJ, 294, 425 1988, ApJ, 330, 445

Skilling, J. 1975, MNRAS, 172, 557

Sreenivasan, S. R., \& Fichtner, H. 2001, in COSPAR Colloq. 11, The Outer Heliosphere: The Next Frontiers, ed. K. Scherer et al. (Amsterdam: Pergamon), 207

Steenberg, C. D., \& Moraal, H. 1996, ApJ, 463, 776

Wang, C., \& Richardson, J. D. 2003, J. Geophys. Res., 108, 1058
Zank, G. P. 1999, Space Sci. Rev., 89, 413

Zank, G. P., Axford, W. I., \& McKenzie, J. F. 1990, A\&A, 233, 275

Zank, G. P., Matthaeus, W. H., Bieber, J. W., \& Moraal, H. 1998, J. Geophys. Res., 103, 2085

Zank, G. P., Matthaeus, W. H., \& Smith, C. W. 1996a, J. Geophys. Res., 101, 17093

Zank, G. P., Pauls, H. L., Cairns, I. H., \& Webb, G. M. 1996b, J. Geophys. Res., 101, 457

Zank, G. P., Rice, W. K. M., le Roux, J. A., Cairns, I. H., \& Webb, G. M. 2001a, Phys. Plasmas, 8, 4560

Zank, G. P., Rice, W. K. M., le Roux, J. A., \& Matthaeus, W. H. 2001b, ApJ, 556,494

Zank, G. P., Webb, G. M., \& Donohue, D. J. 1993, ApJ, 406, 67 